lysate are digested. Using tandem MS, researchers can compare the levels of native and synthetic peptides in a cell to obtain a measure of the amount of native proteins present. Synthetic peptides can also be prepared with modifications, such as extra phosphate groups, to measure the number of post-translationally modified proteins. "We are pursuing the dynamics of protein networks by quantifying changes in the amount of proteins present in specific protein complexes," says Harper. "Techniques such as AQUA provide an accurate and sensitive measure of how the stoichiometry of components within complexes that make up a network are altered in response to a stimulus."

The team used the approach to describe the rearrangements that occur in the protein network of cullin-RING ubiquitin ligases, enzymes that regulate protein turnover, under various cellular conditions ${ }^{11}$.

\section{DEVELOPMENTS IN DIAGNOSTICS}

Changes in protein-protein interaction networks may provide information about the mechanisms of disease. Last year, Wrana applied the network approach to the diagnosis of breast cancer. He used microarrays to measure genome-wide protein expression in the tumours of people with breast cancer, and then overlaid the expression data on the network diagram of the human interactome.

Wrana had noted that 'hub' proteins, defined as those that interact with more than four others, can be grouped into two categories depending on whether they are expressed at the same time as the proteins with which they interact. When they looked at breast-cancer samples, Wrana and his colleagues found that
GENEMANIA

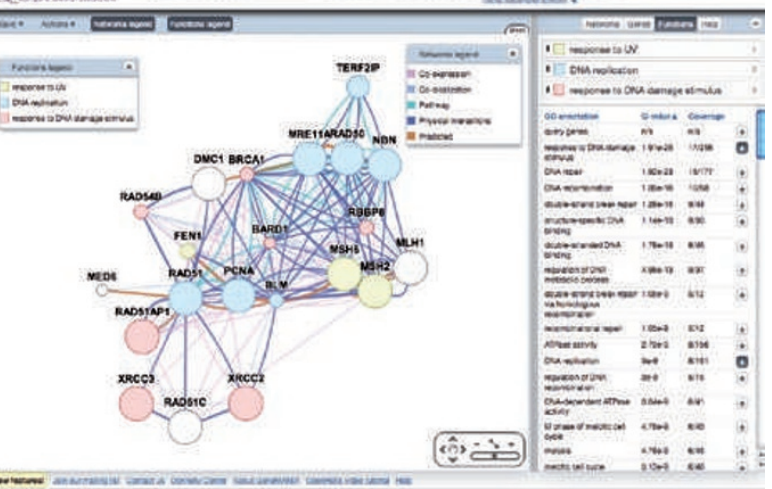

Web tools such as GeneMANIA integrate data on a protein or gene.

develop the method ${ }^{12}$.

According to Gygi, the biggest application of KAYAK might be in tumour classification. "Biopsies or excised tissues can be profiled for kinase activities with pinpoint accuracy. These patterns could contribute towards personalized drug treatments based on dysregulated kinase pathways," he says.

The combination of different types of data and technologies should continue to fill in the empty spaces of the current human interactome map. The picture may never be complete, but it will continue to provide insights into cellular mechanisms of health and disease. "I think that the network

certain hub proteins were in a different category in breast-cancer patients with a good prognosis than in those with a poor prognosis.

Thus, by overlaying the expression pattern of a cancer cell from an individual patient onto the human interactome network, Wrana could predict a patient's prognosis. "We found that the detection of global changes in network organization is more predictive of outcome than is gene expression alone," says Wrana. "We have now applied this method to other tumour models and obtained similar results."

KAYAK (kinase activity assay for protein profiling) is another approach to developing diagnostic tools for cancer on the basis of the functional consequences of the interaction between a protein, in this case a kinase, and its substrate. In this method, up to 90 peptide substrates for kinases are used to simultaneously measure the addition of phosphate groups to proteins in a cell lysate - in essence providing a 'phosphorylation signature' for that particular cell. "The readout is so sensitive and so quantitative that even small differences are teased out," says Gygi, who helped to we have is dense enough for us to start doing studies to classify disease states," says Wrana. "As the networks become better and coverage improves, the accuracy of diagnosis will also improve."

\section{Laura Bonetta is a freelance science writer} based in Garrett Park, Maryland.

1. Venkatesan, K. et al. Nature Meth. 6, 83-90 (2009).

2. Fields, S. \& Song, O.-K. Nature 340, 245-246 (1989).

3. van den Berg, D. L. C. et al. Cell Stem Cell 6, 369-381 (2010).

4. Pardo, M. et al. Cell Stem Cell 6, 382-395 (2010).

5. Sowa, M. E., Bennett, E. J., Gygi, S. P. \& Harper, J. W. Cell 138, 389-403 (2009).

6. Behrends, C., Sowa, M. E., Gygi, S. P. \& Harper, J. W. Nature 466, 68-76 (2010).

7. Bandyopadhyay, S. et al. Nature Meth. 7, 801-805 (2010).

8. Nomura, D. K., Dix, M. M. \& Cravatt, B. F. Nature Rev. Cancer 10, 630-638 (2010).

9. Weerapana, E. et al. Nature 468, 790-795 (2010).

10.Barrios-Rodiles, M. et al. Science 307, 1621-1625 (2005).

11.Bennett, E. J. et al. Cell (in the press).

12. Yonghao, Y. et al. Proc. Natl Acad. Sci. USA 106, 11606-11611 (2009)

13.Uemura, S. et al. Nature 464, 1012-1017 (2010)

\title{
Real-time analysis
}

In November, Pacific Biosciences of Menlo Park, California, commercially released its third-generation DNA-sequencing platform, based on its single-molecule, real-time (SMRT) technology. A single DNA polymerase bound to a DNA template is attached to a tiny chamber illuminated by lasers, and nucleotides labelled with coloured fluorophores are introduced to it. As the polymerase incorporates them, each base is held for a few microseconds, while the fluorophore emits coloured light corresponding to the base identity. SMRT technology could also be used to analyse biomolecules other than DNA, and could become a common tool for detecting protein interactions, with some unique features. "This technology can detect relatively weak interactions," says Jonas Korlach, a scientific fellow at Pacific Biosciences, adding that it could pick out interactions that happen so quickly that they can't be identified by current methods.

As a step towards such applications, Joseph Puglisi, a structural biologist at Stanford University School of Medicine in California, and his group, with scientists at Pacific Biosciences, observed transfer RNAs binding to single ribosomes in real time ${ }^{13}$. In an unpublished follow-up, Puglisi's group has used SMRT technology to watch interactions between transfer RNAs, ribosomes and

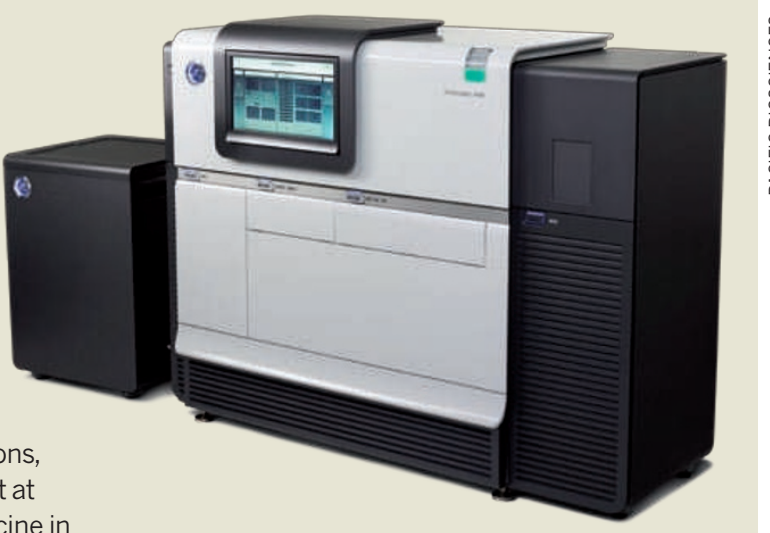

Future SMRT systems could reveal interactions.

protein factors to determine how the translation machinery synthesizes proteins. "We have just seen the tip of the iceberg in terms of applications," says Korlach. L.B. 